OPEN ACCESS

Edited by:

Olivier Feron,

Université catholique de Louvain,

Belgium

Reviewed by:

Ellen Smith,

University of Alabama at Birmingham,

United States

Lorenzo Di Cesare Mannelli,

University of Florence, Italy

*Correspondence:

Aishwarya Bandla

aishwarya.bandla@nus.edu.sg

Yu Yang Soon

yu_yang_soon@nuhs.edu.sg

${ }^{t}$ These authors have contributed equally to this work

Specialty section:

This article was submitted to Pharmacology of Anti-Cancer Drugs,

a section of the journal

Frontiers in Oncology

Received: 28 June 2021 Accepted: 10 January 2022 Published: 03 February 2022

Citation:

Peng S, Ying AF, Chan NJH, Sundar $R$, Soon $Y Y$ and Bandla $A$

(2022) Prevention of Oxaliplatin-

Induced Peripheral Neuropathy: A Systematic Review and Meta-Analysis.

Front. Oncol. 12:731223.

doi: 10.3389/fonc.2022.731223

\section{Prevention of Oxaliplatin-Induced Peripheral Neuropathy: A Systematic Review and Meta-Analysis}

\author{
Siyu Peng ${ }^{1 \dagger}$, Ariel Fangting Ying ${ }^{2 \dagger}$, Nicholas Jian Hao Chan ${ }^{1}$, Raghav Sundar ${ }^{3,4,5}$, \\ Yu Yang Soon ${ }^{6 *}$ and Aishwarya Bandla ${ }^{3,4 *}$ \\ ${ }^{1}$ Department of Medicine, National University Health System, Singapore, Singapore, ${ }^{2}$ Health Services and System Research, \\ Duke-National University of Singapore (NUS) Medical School, Singapore, Singapore, ${ }^{3}$ Department of Haematology- \\ Oncology, National University Cancer Institute Singapore, National University Health System, Singapore, Singapore, ${ }^{4}$ The \\ N. 1 Institute for Health, National University of Singapore, Singapore, Singapore, 5 Yong Loo Lin School of Medicine, National \\ University of Singapore, Singapore, Singapore, ${ }^{6}$ Department of Radiation Oncology, National University Cancer Institute \\ Singapore, National University Health System, Singapore, Singapore
}

Background: Oxaliplatin-induced peripheral neuropathy (OIPN) has significant clinical impact on the quality of life for cancer patients and is a dose limiting toxicity. Trials studying preventive measures have been inconclusive. A systematic review and meta-analysis were conducted to evaluate the existing pharmacological and non-pharmacological interventions to prevent chronic OIPN.

Methods: Literature databases PubMed-MEDLINE, Embase and Scopus, were searched from 1 Jan 2005 to 08 Aug 2020 and major conferences' abstracts were reviewed for randomized controlled trials that examined the efficacy of any preventive measure for OIPN. The primary outcome measure was the incidence of chronic OIPN with a preventive intervention as compared to placebo or no intervention. The pooled risk ratio and its 95\% confidence interval were calculated using a random effects model. A network meta-analysis was conducted to derive indirect evidence of any preventive effect of an intervention against placebo when original trials compared one intervention against another.

Results: Forty-four trials were analyzed describing 29 chemoprotective interventions, including combinations, and 1 non-pharmacological intervention. Ratings were assessed via a combination of outcomes with quality assessment using the Grading of Recommendations, Assessment, Development and Evaluations (GRADE) framework. Of the 30 interventions examined, there were six interventions supporting potential efficacy, 11 interventions with insufficient evidence and 13 interventions not recommended.

Conclusion: Currently, there is insufficient certainty to support any intervention as effective in preventing OIPN. Of note is that most of these studies have focused on pharmacological interventions; non-pharmacological interventions are underexplored. Further research on ways to limit OIPN is needed. 


\section{Systematic Review Registration: https://www.crd.york.ac.uk/prospero/display_ record.php?RecordID=225095, Prospero Registration Number: CRD42021225095.}

Keywords: oxaliplatin, peripheral neuropathy, neurotoxicity, meta-analysis, pharmacological, non-pharmacological, network analysis

\section{INTRODUCTION}

Oxaliplatin, a platinum-based anti-tumor agent, is a mainstay of treatment for many gastrointestinal cancers, particularly colorectal cancer, the second highest cause of cancer mortality worldwide (1). However, as many as $40-50 \%$ of patients receiving this drug will develop oxaliplatin-induced peripheral neuropathy (OIPN) $(2,3)$. While the acute form of OIPN is largely reversible within a week, about $20-50 \%$ of patients experience severe chronic OIPN which is dosedependent, potentially persistent and debilitating (4-6). This high incidence of chronic neurotoxicity has significant impact on treatment continuity and quality of life in cancer patients and survivors, making OIPN an area of significant clinical importance $(7,8)$.

Many mechanisms for OIPN have been postulated, ranging from direct neurotoxicity from deoxyribonucleic acid (DNA) damage to ion channel dysfunction to oxidative stress $(9,10)$. As such, pre-clinical studies on OIPN prevention have examined the efficacy of a wide range of potential interventions, from neuroactive pharmaceuticals like gabapentin (11) and antioxidants like glutathione (12), to herbal medications like Goshajinkigan (13). However, attempts to translate encouraging results from animal studies to humans have met with limited success.

Although many clinical trials have attempted to identify interventions that can prevent OIPN in humans, the small sample size in most of these trials and the lack of standardized measurement metrics have rendered results difficult to interpret (14). Indeed, the latest oncology guidelines on chemotherapyinduced peripheral neuropathy (CIPN) acknowledge that despite the multitude of trials available, there is no convincing evidence that there is any intervention that can effectively prevent CIPN $(7,8)$.

In recent years, there has been an emerging interest in examining the usage of non-pharmacological interventions to prevent CIPN, such as cryotherapy, acupuncture, or exercise $(15-18)$. One area that has drawn particular interest is the usage of cryotherapy, which has yielded promising results in taxaneinduced peripheral neuropathy $(16,19,20)$. Subsequent trials have recently begun looking into the efficacy of cryotherapy on OIPN as well (21).

Previous systematic reviews and meta-analyses have attempted to synthesize all the information available on CIPN, but they either focused on one specific intervention $(22,23)$ and/ or were not specific to oxaliplatin $(7,8,24)$. Given that there are distinct differences in the mechanism and clinical presentation of neuropathy induced by different chemotherapy agents, and between acute and chronic OIPN, evidence that is specific to chronic OIPN might better inform clinical practice $(7,25,26)$.
This current systematic review/meta-analysis was conducted to determine the efficacy of both pharmacological and nonpharmacological preventive measures for chronic OIPN in patients with cancers treated with oxaliplatin-based chemotherapy.

\section{METHODS}

\section{Search Strategy}

The systemic review and meta-analysis followed the Preferred Reporting Items for Systematic Reviews and Meta-analyses (PRISMA) guidelines (27). Two investigators (SP and AFY) independently searched, without language restriction, PubMedMEDLINE, Embase and Scopus for randomized controlled trials (RCTs) published since 1 Jan 2005 to 8 Aug 2020. A detailed list of the search terms can be found in the Supplementary Material.

\section{Selection Criteria}

Human RCTs that assessed the efficacy of any form of protective measure to try to prevent and/or reduce the incidence of chronic OIPN as compared with placebo, no intervention or other interventions were included. These trials must have enrolled adult cancer patients with the planned chemotherapy regimen containing oxaliplatin as the only neurotoxic chemotherapeutic agent and reported the incidence and/or severity of chronic OIPN measured using clinical scales or electrophysiological studies. Trials were excluded if they were single-armed, retrospective, non-randomized, enrolled patients with preexisting peripheral neuropathy, enrolled patients not naïve to chemotherapy, did not report outcomes specific to OIPN, or only reported acute OIPN outcomes. The primary outcome was the incidence of grade 2 and above peripheral neuropathy as assessed by the Common Terminology Criteria for Adverse Events (CTCAE). Information on other outcomes such as neuropathy grades assessed by other neuropathy scales or electrophysiology measurements were also extracted. For trials that did not use CTCAE in the outcome assessment, the primary outcome measures reported by trials were used for analysis. For the trials that did not report the full details of outcomes for analysis, the authors were contacted for more information. If such data were not available, then such trials were excluded from analysis.

\section{Data Extraction}

The same investigators (SP and AFY) independently extracted data from the selected trials. Discrepancies were resolved by consensus amongst both investigators and advice from a third independent investigator (RS). Information extracted included: intervention dose and route, nature of the control (placebo, 
no additional intervention, or another intervention), trial primary and secondary outcomes and their assessment methods (clinical neuropathy scales or neurological electrophysiological studies) and time of assessment, follow up duration, and cumulative dose of oxaliplatin. Information was also collected for trial quality assessment, as outlined below.

\section{Quality Assessment}

Quality of the trials was assessed using the Risk of Bias Tool in Review Manager version 5.3 (RevMan 5.3) software by Nordic Cochrane Centre, and scored according to the domains of selection bias, performance bias, detection bias, attrition bias and reporting bias. The risk of bias results were scored on three levels, namely "high bias", "low bias" and "unclear bias", and then included into a rating approach proposed by the Grading of Recommendations, Assessment, Development and Evaluation (GRADE) Working Group to evaluate the certainty of the result across four domains, namely risk of bias, inconsistency, indirectness and imprecision (28). The final GRADE scores represent four levels of certainty, namely "very low" (1), "low" (2), "moderate" (3) and "high" (4) (Supplementary Material).

\section{Data Analysis}

For trials using dichotomous outcome measures, the treatment effect was calculated across trials in the form of Risk Ratio (RR) based on the intention-to-treat sample size. If there were two or more RCTs that reported the same outcome measure by comparing the same combination of interventions, the respective $\mathrm{RR}$ were summarized in Forest plots using the random effects model of meta-analysis, in RevMan 5.3. All reported $p$-values were two-sided and $p<0.05$ indicated statistical significance. Heterogeneity was assessed via Cochran's Q test and Higgins' $\mathrm{I}^{2}$ test. If significant methodological or statistical heterogeneity was detected, sensitivity analysis was performed based on factors that contributed to heterogeneity. For continuous outcomes, such as pain or neuropathy scores, the difference in means was calculated and the results were expressed with 95\% confidence intervals (CI). For outcomes that did not fit the above criteria, significant outcomes were summarized as they were presented in the results section of the original trial.

A network meta-analysis was also conducted using $\mathrm{R}$ programming (netmeta package, frequentist method and random effects model), to obtain indirect evidence of treatment effect from the trials that compared two types of interventions. The main purpose of performing a network meta-analysis is to be able to derive indirect comparisons between a type of intervention and control (either placebo or no additional intervention), as such information was not available from the original trials (29).

\section{RESULTS}

\section{Trial Selection}

The search strategy (Figure 1) derived a total of 44 RCTs that examined 30 interventions for OIPN prevention, of which 43

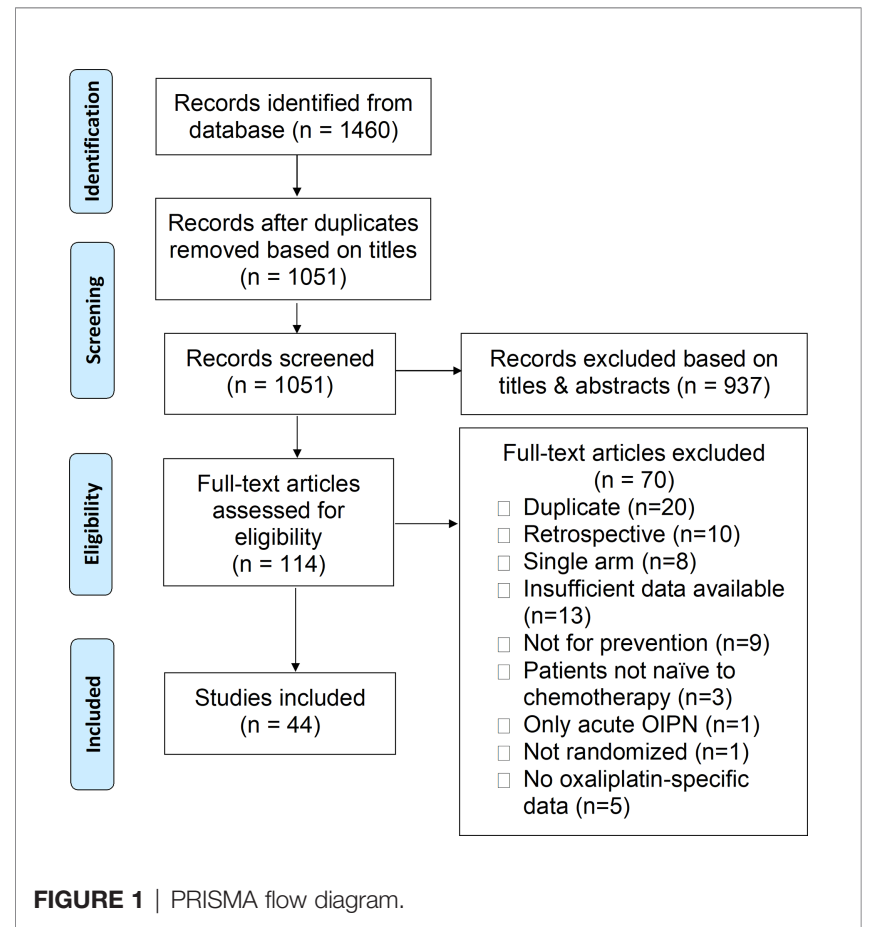

trials examined 29 pharmacological interventions and 1 trial examined 1 non-pharmacological interventions. Of those that compared pharmacological interventions against placebo or no additional intervention, seven trials examined calcium and magnesium $(\mathrm{Ca} / \mathrm{Mg})$ infusions, ten examined neuro-active pharmaceuticals, six examined other pharmaceuticals, eleven examined antioxidants, and seven examined herbal medicines. One of the trials, Dong et al. (30), assessed two different types of interventions; thus, it was classified under two sections. In addition, three trials compared two types of interventions (or combination interventions) and their results were analyzed separately using network meta-analysis.

Among the excluded studies, seven RCTs that enrolled patients receiving oxaliplatin, and whose primary outcome was the prevention of chronic CIPN, were also identified via full text screening. Of these, two trials $(31,32)$ enrolled patients with prior platinum exposure and the remaining five $(21,33-36)$ did not provide sufficient data for analysis. Hence, all seven of these trials were excluded from the final analysis.

\section{Rating Criteria for Interventions}

Based on the RR calculated from primary outcome measures and the GRADE evaluation of certainty of evidence, the interventions were assigned to one of three levels of recommendation, namely "Has Potential", "Insufficient Evidence" and "Not Recommended" (Figure 2). An intervention was rated to have "Potential" if the RR $<1.00$, the CI did not cross 1.00 , and the GRADE evaluation score was $\geq 3$ ("moderate" or "high"). An intervention was rated to have "Insufficient Evidence" if the RR $<1.00$, but either the CI crossed 1.00 or the GRADE evaluation score was $=2$ ("low"). An intervention was rated to be "Not Recommended" if the RR > 1.00 or the GRADE evaluation score was = 1 ("very low"). 


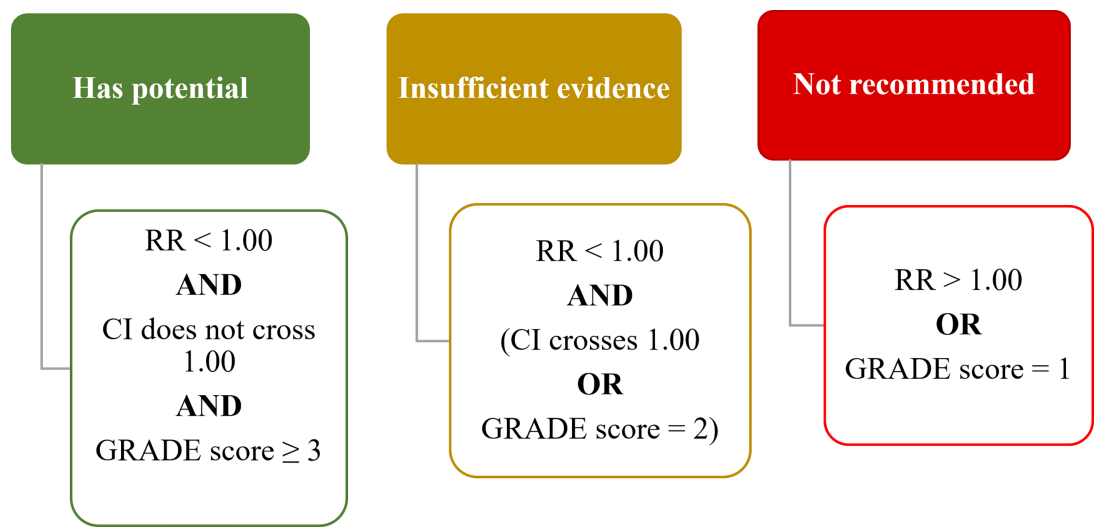

FIGURE 2 | Rating criteria on interventions to prevent OIPN. RR, risk ratio; Cl, confidence interval. GRADE score ranges from High (4+), Moderate (3+), Low (2+) to Very Low (1+). (RR, Risk Ratio; Cl, Confidence Interval).

When there was more than one outcome measure reported, the primary outcome measures using CTCAE was prioritized in the overall evaluation, while evaluations based on other outcome measures were recorded in the Table $\mathbf{S 1}$ and the scales explained in Table S2. In summary, six interventions were rated as having "Potential", nine interventions as "Insufficient Evidence" and fifteen interventions as "Not Recommended".

In addition, among the fourteen interventions with statistically significant lower RR of OIPN incidence, including L-carnosine (Figure S1), six were classified as "insufficient evidence" while two were classified as "not recommended" as their overall ratings were limited by "low" and "very low" GRADE certainties respectively (Table 1).

Refer to the Supplementary Material for summaries of trial characteristics and risk of bias of all trials (Table S3), dichotomous or continuous outcome results analyzed from single trials (Tables S4, S5) and table presentation of network meta-analysis results (Table S6).

\section{Type of Intervention}

For the ease of presentation, the pharmacological interventions were classified into five groups based on mechanisms of action, namely $\mathrm{Ca} / \mathrm{Mg}$, neuro-active pharmaceuticals, other pharmaceuticals, antioxidants, and herbal medicine.

\section{Calcium and Magnesium (Ca/Mg)}

A total of seven trials examined the effect of intravenous $\mathrm{Ca} / \mathrm{Mg}$ infusions versus placebo on the prevention of $\operatorname{OIPN}(3,30,38-$ $41,75)$. Of these, only Gobran et al. (40) reported a statistically significant reduction in OIPN incidence using Chi-square test.

All seven trials were double-blinded and used placebo saline infusions for the control group (Table S3). However, three of the trials, namely the ones by Ishibashi et al. (39), Chay et al. (75), and Grothey et al. (41), were terminated early due to an interim report from the Combined Oxaliplatin Neurotoxicity Prevention Trial (CONcePT) (76) in 2007, which suggested that $\mathrm{Ca} / \mathrm{Mg}$ infusion could worsen response to chemotherapy. In addition, two of the other trials, by Gobran et al. (40) and Dong et al. (30), had high attrition rates of $\geq 30 \%$. Furthermore, the trial by Dong et al. (30) only reported the incidence of neuropathy at each assessment point, instead of the cumulative incidence.

Six of the trials used CTCAE to assess outcome and their results were pooled in a Forest plot (Figure 3) (3, 30, 38-41). Ca/ $\mathrm{Mg}$ infusion was associated with a statistically significant reduction in the risk of CTCAE grade 2 and above neuropathy (RR, 0.74; 95\% CI, 0.55-0.99). However, after performing sensitivity analysis to exclude the two trials with early termination $(39,41)$, pooled results were no longer statistically significant (RR, 0.76; 95\% CI, 0.54-1.08) (Figure S2). Pooled analysis from three trials $(3,41,75)$ on the secondary outcome also showed non-statistically significant reduction in the incidence of OIPN of Oxaliplatin Specific Scale (OSS) grade $\geq$ 2 (RR, 0.76; 95\% CI, 0.56-1.03) (Figure S3). Symmetrical funnel plot (Figure S4) showed low publication bias.

\section{Neuro-Active Pharmaceuticals}

A total of ten trials examined the effect of neuro-active pharmaceuticals versus placebo on the prevention of OIPN. Of these, four of them studied anti-epileptics (carbamazepine, oxcarbazepine, pregabalin and riluzole) $(48,55,56,71)$, four studied pain medications (ganglioside-monosialic acid, Novel Sigma-1 Receptor Antagonist MR309, and neurotropin) (53, 66, 70, 77), and the remaining two studied the antidepressant venlafaxine $(57,58)$ (Table S3).

None of the neuro-active pharmaceuticals showed potential benefit (Table 1).

\section{Other Pharmaceuticals}

A total of six trials examined the effect of other pharmaceuticals versus placebo on the prevention of OIPN. There were two trials on $\mathrm{N}$-acetylcysteine $(46,47)$, one on calmangafodipir $(63)$, one on metformin (52), one on minocycline (69), and one on thrombomodulin (62) (Table S3).

Only $\mathrm{N}$-acetylcysteine showed potential for OIPN prevention (Table 1). Pooled results suggested an association between $\mathrm{N}$ acetylcysteine and a reduced risk of CTCAE grade 2 and above 


\begin{tabular}{|c|c|c|c|c|c|c|c|c|c|}
\hline Intervention & Outcome & No. of studies & No. of patients & RR (95\% Cl) & Certainty & Risk of bias & Inconsistency & Indirectness & Imprecision \\
\hline \multicolumn{10}{|c|}{ Has Potential } \\
\hline AC591 (37) & Levi >= Grade 2 & 1 & 72 & RR $0.09(0.01,0.67)^{*}$ & High (4+) & Not serious & Not serious & Not serious & Not serious \\
\hline $\mathrm{Ca} / \mathrm{Mg}(3,30,38-41)$ & $\operatorname{CTCAE}^{\wedge}>=$ Grade 2 & 6 & 613 & RR $0.74(0.55,0.99)^{*}$ & Moderate $(3+)$ & Serious & Not serious & Not serious & Not serious \\
\hline Glutathione (GSH) $(30,42,43)$ & CTCAE $>=$ Grade 2 & 3 & 159 & RR $0.50(0.36,0.72)$ * & High (4+) & Not serious & Not serious & Not serious & Not serious \\
\hline Guilongtongluofang (44) & CTCAE Grade 3-4 & 1 & 120 & $\operatorname{RR} 0.37(0.17,0.81)^{*}$ & High (4+) & Not serious & Not serious & Not serious & Not serious \\
\hline N3 PUFA (45) & Reduced TNS ${ }^{\dagger}$ incidence & 1 & 71 & RR $0.60(0.43,0.83)^{*}$ & High (4+) & Not serious & Not serious & Not serious & Not serious \\
\hline N-acetylcysteine (NAC) $(46,47)$ & CTCAE >= Grade 2 & 2 & 46 & RR $0.26(0.12,0.56)$ * & Moderate (3+) & Serious & Not serious & Not serious & Not serious \\
\hline \multicolumn{10}{|c|}{ Insufficient Evidence } \\
\hline Carbamazepine (48) & Levi Grade 3-4 & 1 & 36 & RR $0.60(0.20,1.76)$ & Low (2+) & Serious & Not serious & Not serious & Serious \\
\hline Glutamine (49) & CTCAE Grade 3-4 & 1 & 86 & RR $0.37(0.15,0.95)^{*}$ & Low $(2+)$ & Very serious & Not serious & Not serious & Not serious \\
\hline L-carnosine $(50,51)$ & CTCAE $>=$ Grade 2 & 2 & 121 & RR $0.05(0.01,0.22)$ * & Low (2+) & Very serious & Not serious & Not serious & Not serious \\
\hline Metformin (52) & CTCAE $>=$ Grade 2 & 1 & 40 & RR $0.63(0.44,0.92)$ * & Low $(2+)$ & Very serious & Not serious & Not serious & Not serious \\
\hline Neurotropin (53) & CTCAE $>=$ Grade 2 & 1 & 79 & RR $0.35(0.18,0.67)^{*}$ & Low $(2+)$ & Very serious & Not serious & Not serious & Not serious \\
\hline Ninjin'yoeito (54) & CTCAE $>=$ Grade 2 & 1 & 52 & RR $0.18(0.04,0.74)^{*}$ & Low (2+) & Very serious & Not serious & Not serious & Not serious \\
\hline Oxcarbazepine (55) & Modified TNS ${ }^{\dagger}$ incidence & 1 & 40 & RR $0.42(0.18,0.96)$ * & Low (2+) & Serious & Not serious & Not serious & Not serious \\
\hline Pregabalin (56) & Brief pain inventory & 1 & 143 & $\begin{array}{l}\text { Continuous, non-significant } \\
\text { benefit }\end{array}$ & Low $(2+)$ & Serious & Not serious & Not serious & Serious \\
\hline Venlafaxine $(57,58)$ & CTCAE $>=$ Grade 2 & 2 & 101 & RR $0.75(0.47,1.19)$ & Moderate $(3+)$ & Not serious & Not serious & Serious & Serious \\
\hline \multicolumn{10}{|c|}{ Not Recommended } \\
\hline Air wave pressure (59) & CTCAE $>=$ Grade 2 & 1 & 45 & RR $0.19(0.02,1.51)$ & Very low (1+) & Very serious & Not serious & Not serious & Serious \\
\hline Alpha-lipoic acid (ALA) (60) & $\mathrm{NCl}$ Sanofi ${ }^{\ddagger}>=$ Grade 2 & 1 & 49 & RR $0.69(0.39,1.23)$ & Very low (1+) & Very serious & Not serious & Not serious & Serious \\
\hline Amifostine $(49,61)$ & CTCAE Grade 3-4 & 2 (NMA) & 173 & RR $0.02(0.00,0.48)^{*}$ & Very low (1+) & Serious & Not serious & Serious & Serious \\
\hline ART-123 (62) & CTCAE >= Grade 2 & 1 & 42 & RR $1.40(0.84,2.35)$ & Low $(2+)$ & Serious & Not serious & Not serious & Serious \\
\hline Calmangafodipir (63) & OSSS ${ }^{\ddagger}$ Grade 2-3 & 1 & 173 & RR $0.61(0.32,1.16)$ & Very low (1+) & Very serious & Not serious & Serious & Serious \\
\hline Cystine \& theanine (64) & Mean CTCAE score & 1 & 28 & $\begin{array}{l}\text { Continuous, reported as } \\
\text { significant benefit * }\end{array}$ & Very low (1+) & Very serious & Not serious & Serious & Not serious \\
\hline Glutamine + Ca/Mg (3, 30, 38-41, 65) & CTCAE >= Grade 2 & 7 (NMA) & 813 & RR $0.76(0.43,1.35)$ & Very low (1+) & Very serious & Not serious & Serious & Serious \\
\hline Ganglioside-monosialic acid (GM1) (66) & CTCAE $>=$ Grade 2 & 1 & 192 & RR $1.07(0.71,1.61)$ & Moderate (3+) & Not serious & Not serious & Not serious & Not serious \\
\hline Goshajinkigan (GJG) $(67,68)$ & CTCAE $>=$ Grade 2 & 2 & 271 & RR $1.12(0.53,2.37)$ & Very low (1+) & Serious & Serious & Not serious & Serious \\
\hline Minocycline (69) & MDASI AUC $\#$ & 1 & 66 & Continuous, non-significant harm & Moderate $(3+)$ & Not serious & Not serious & Not serious & Serious \\
\hline MR309 (70) & CTCAE $>=$ Grade 2 & 1 & 87 & $\operatorname{RR} 1.18(0.91,1.52)$ & Low $(2+)$ & Serious & Not serious & Serious & Serious \\
\hline Riluzole (71) & Reduced TNS ${ }^{\dagger}$ incidence & 1 & 48 & Continuous, significant harm & High (4+) & Not serious & Not serious & Not serious & Not serious \\
\hline Tanshinone IIA (72) & Levi's Grade 3-4 & 1 & 36 & RR $5.00(0.26,97.37)$ & Very low (1+) & Very serious & Not serious & Not serious & Serious \\
\hline Vitamin E (73) & Neuropathy incidence & 1 & 65 & RR $1.03(0.95,1.12)$ & Very low (1+) & Very serious & Not serious & Not serious & Serious \\
\hline Vitamin E + Ca/Mg (3, 30, 38-41, 74) & CTCAE $>=$ Grade 2 & 7 (NMA) & 647 & RR $1.98(0.22,17.95)$ & Very low (1+) & Not serious & Not serious & Serious & Serious \\
\hline
\end{tabular}

Total 30 interventions were classified into three levels of recommendation, namely "have potential", "insufficient evidence and "not recommended".

CTCAE^, Common Terminology Criteria for Adverse Events; TNS ${ }^{\dagger}$, Total Neuropathy Score; NCI Sanofi/OSSS", National Cancer Institute Sanofi scale, also known as Oxaliplatin Sanofi Specific Scale; MDASI AUC ${ }^{\sharp}$, MD Anderson Symptom Inventory (MDASI) score for numbness/tingling and fatigue, calculated as Area Under Curve; Risk ratios marked with * indicates that they are statistically significant where $95 \%$ confidence interval is less than 1. 


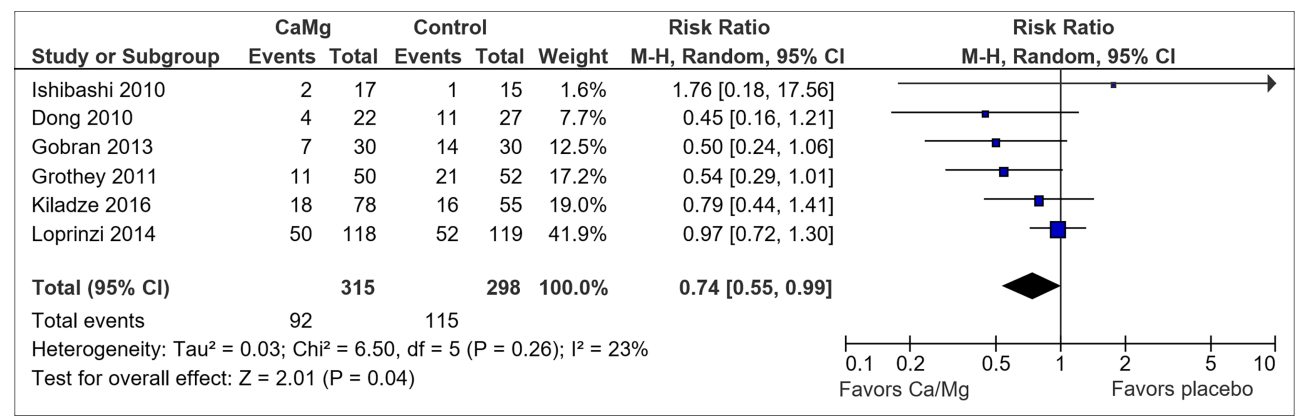

FIGURE 3 | Forest plot for comparison of the incidence of OIPN of CTCAE grade $\geq 2$ : Ca/Mg versus placebo.

neuropathy (RR, 0.26; 95\% CI, 0.12-0.56) (Figure 4). However, the pooled sample size is small $(\mathrm{n}=46)$. In addition, the trial by Lin et al. (46) did not utilize blinding or placebo and hence further studies are warranted.

\section{Antioxidants}

A total of eleven trials examined the effect of antioxidants versus placebo on the prevention of OIPN. These included one trial on alpha lipoic acid (60), two on glutamine $(49,78)$, three on reduced glutathione $(30,42,43)$, two on L-carnosine $(50,51)$, one on vitamin $\mathrm{E}$ (73), one on $\mathrm{n}-3$ polyunsaturated fatty acids (n3 PUFA) (45), and one on cystine plus theanine (64) (Table S3).

Reduced glutathione was assessed to have potential benefit in preventing OIPN (Table 1). Pooled results from the three trials on reduced glutathione $(30,42,43)$ suggested an association between reduced glutathione and a lowered risk of CTCAE grade
2 and above neuropathy (RR, 0.50; 95\% CI, 0.36-0.72) (Figure 5). The overall certainty of the trials was high. However, the trial by Dong et al. (30) had a high attrition rate of $47.3 \%$ by cycle $9(20 \%$ by cycle 6). The trial by Milla et al. (42) and Pang et al. (43) used placebo but did not mention if allocation concealment or blinding was utilized.

N-3 PUFA also showed potential benefit in preventing OIPN. The only trial on n-3 PUFA by Esfahani et al. (45) showed a reduction in the risk of neuropathy as measured by the reduced Total Neuropathy Score (RR, 0.60; 95\% CI, 0.430.83) (Table 1). However, this benefit was not consistently observed in secondary outcome measured in sensory nerve conduction studies and the trial was limited by the suboptimal placebo use (placebo did not smell like the intervention), small sample size and short follow-up period, thus requiring further studies to affirm the result.

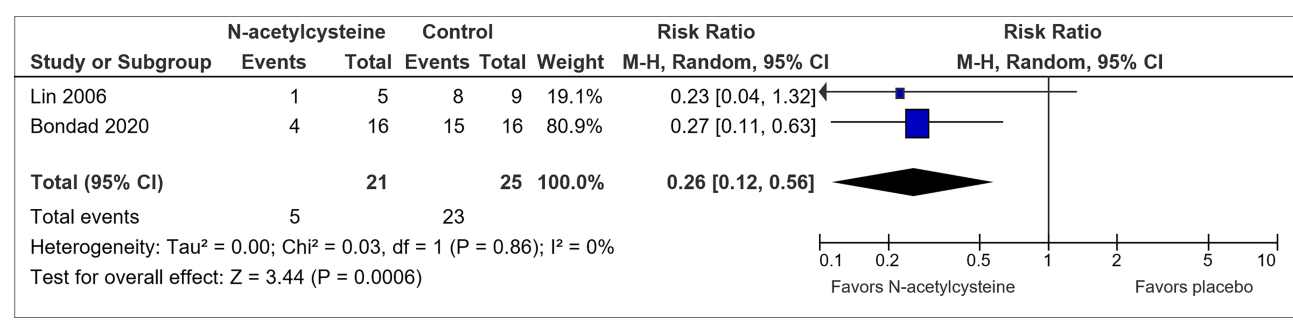

FIGURE 4 | Forest plot for comparison of the incidence of OIPN of CTCAE grade $\geq 2$ : N-acetylcysteine versus placebo.

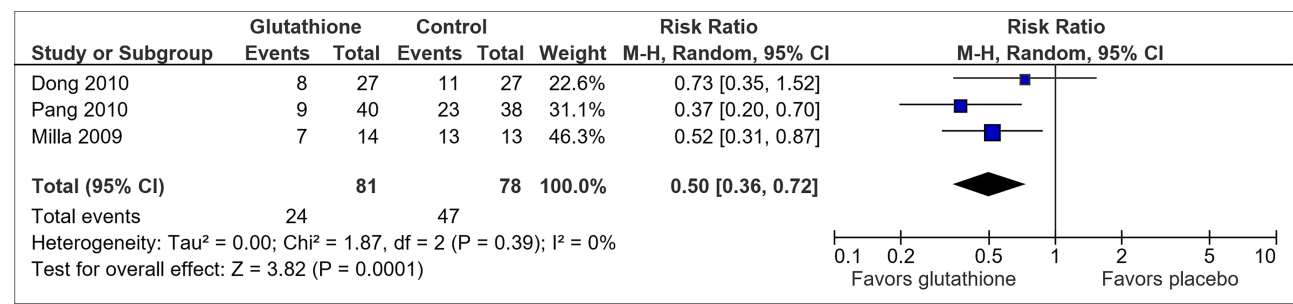

FIGURE 5 | Forest plot for comparison of the incidence of OIPN of CTCAE grade $\geq 2$ : Reduced glutathione versus placebo. 


\section{Herbal Medicines}

A total of seven trials examined the effect of herbal medicines versus placebo on the prevention of OIPN. There were three trials on goshajinkigan $(67,68,79)$, one on Guilongtongluofang (44), one on ninjin'yoeito (54), one on Tanshinone IIA (72), and one on AC591 (37) (Table S3). Only AC591 and guilongtongluofang were rated to "have potential" in reducing OIPN risk (Table 1).

AC591 was associated with a reduction in grade 2 and above neuropathy assessed via Levi's scale (RR, 0.09; 95\% CI, 0.01-0.67) (37). However, participants were only followed up for two months, i.e., four cycles of chemotherapy.

Guilongtongluofang was associated with a reduction in CTCAE grade 3-4 neuropathy (RR, 0.37; 95\% CI, 0.17-0.81) (44). This trial had a low risk of bias in all categories. Patients received six cycles of chemotherapy and were followed up for two months thereafter.

\section{Non-Pharmacological Interventions}

Only one trial examined the effect of non-pharmacological interventions on the prevention of OIPN (Table S3). In their trial on air wave pressure, Qian et al. (59) did not find any association with OIPN incidence (RR, 0.19; 95\% CI, 0.02-1.51) (Table 1). Due to the nature of the intervention, placebo usage and thus blinding was not possible.

\section{Network Meta-Analysis}

In order to assess treatment effect in the trials that compared two different interventions or intervention combinations, network meta-analysis was performed to link these trials with other related trials. There were three such trials $(61,65,74)$ (Table S3). Network graphs (Figure S5) illustrate the type of comparison between different interventions. The presence of lines between a pair of nodes (interventions) indicates direct comparison from original trials, while the absence of a line indicates indirect comparison derived from network metaanalysis (29). The thickness of the lines is proportional to the number of trials that studied the respective direct comparison.
The trial by $\mathrm{Lu}$ et al. assessed the efficacy of amifostine versus glutamine (61). This was linked to a trial by Wang et al., which compared glutamine against a control group that received no additional intervention (49) (Figure S5A). The resultant network analysis (Figure 6) suggested that both amifostine (RR, 0.02; 95\% CI, 0.00-0.48) and glutamine (RR, 0.37 ; $95 \%$ CI, 0.15-0.95) were associated with a reduced risk of Grade 3-4 OIPN as compared to no additional intervention. However, the overall GRADE certainty for the two studies involved was "very low (1+)", and hence amifostine was rated "Not Recommended" (Table 1).

In a network of seven trials (Figure S5B), six trials assessed $\mathrm{Ca} / \mathrm{Mg}$ versus placebo $(3,30,38-41)$ while one trial assessed a combination of glutamine and $\mathrm{Ca} / \mathrm{Mg}$ versus $\mathrm{Ca} / \mathrm{Mg}$ alone (65). The resultant RR comparing the combination of glutamine and $\mathrm{Ca} / \mathrm{Mg}$ versus placebo was not statistically significant $(\mathrm{RR}, 0.76$; 95\% CI, 0.43-1.35) (Figure 7), and the trial by Samson et al. (65) also used an open label design instead of blinding. Hence, the combination of glutamine plus $\mathrm{Ca} / \mathrm{Mg}$ was rated "Not Recommended" (Table 1).

In another network of seven trials (Figure S5C), six trials compared $\mathrm{Ca} / \mathrm{Mg}$ versus placebo $(3,30,38-41)$ while one trial compared the combination of vitamin $\mathrm{E}$ and $\mathrm{Ca} / \mathrm{Mg}$ versus $\mathrm{Ca} /$ $\mathrm{Mg}$ alone (74). This combination of vitamin $\mathrm{E}$ and $\mathrm{Ca} / \mathrm{Mg}$ was also rated "Not Recommended" since the indirect comparison showed RR of 1.98 (95\% CI, 0.22-17.95) between the combination of Vitamin $\mathrm{E}$ and $\mathrm{Ca} / \mathrm{Mg}$ against placebo (Figure 8 and Table 1).

\section{DISCUSSION}

This meta-analysis provides a focused update on existing evidence of OIPN prevention methods, including pharmacological and non-pharmacological interventions. Thirty interventions were analyzed comparing the risk ratio measured using clinical scales or electrophysiological testing. For the ease of presentation, the pharmacological interventions were classified into five
Intervention

Amifostine
Glutamine
No additional intervention

\section{Intervention vs. Control} (CTCAE Grade 3-4)

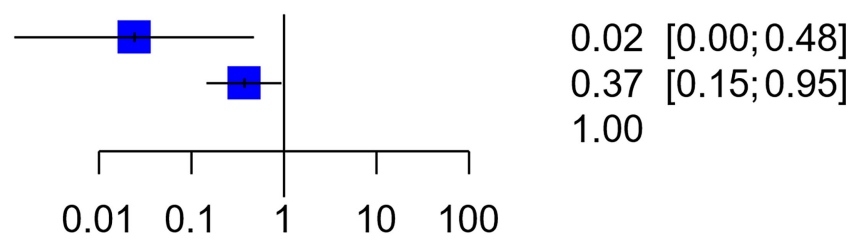

\section{Favors Intervention Favors Control}

FIGURE 6 | Forest plot for network analysis comparing Amifostine, Glutamine and control (no additional intervention), on the risk of CTCAE Grade 3-4 OIPN. Risk ratio comparing amifostine to no additional intervention was derived indirectly from network meta-analysis, while the risk ratio comparing glutamine against no additional intervention was derived directly from original trial data. 


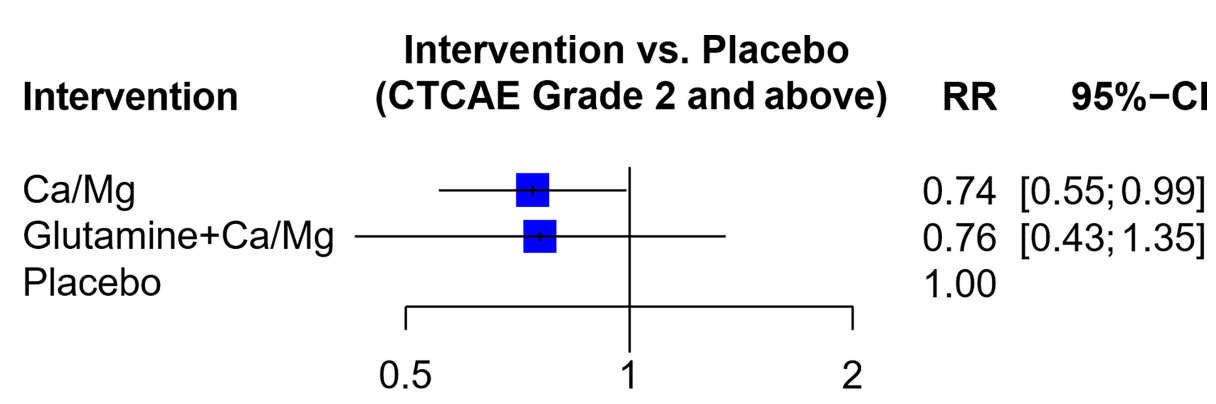

Favors Intervention Favors Placebo

FIGURE 7 | Forest plot for network analysis comparing Glutamine + Ca/Mg, Ca/Mg and placebo on the risk of OIPN of CTCAE grade $\geq 2$. Risk ratio comparing combination therapy "glutamine + Ca/Mg" against placebo was derived indirectly from network meta-analysis, while the risk ratio comparing Ca/Mg against placebo was derived directly from 6 trials that conducted the exact comparison.

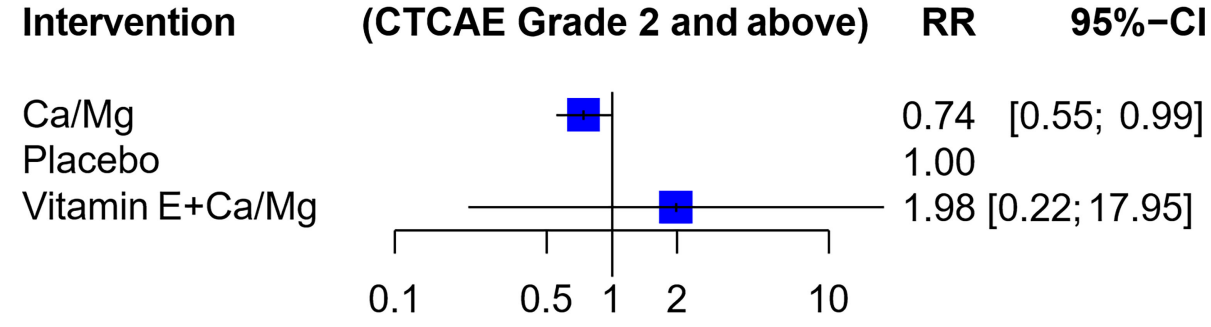

Favors Intervention Favors Placebo

FIGURE 8 | Forest plot for network analysis comparing Vitamin E + Ca/Mg, Ca/Mg and placebo on the risk of OIPN of CTCAE grade $\geq 2$. Risk ratio comparing combination therapy "Vitamin E+ Ca/Mg" against placebo was derived indirectly from network meta-analysis, while the risk ratio comparing Ca/Mg against placebo was derived directly from 6 trials that conducted the exact comparison.

groups, namely $\mathrm{Ca} / \mathrm{Mg}$, neuro-active pharmaceuticals, other pharmaceuticals, antioxidants, and herbal medicine.

Based on outcome measures and the GRADE evaluation of certainty of evidence, the interventions were assigned to one of three levels of recommendation, namely "Has Potential", "Insufficient Evidence" and "Not Recommended" (Figure 2). With these criteria, six interventions that may have potential were identified, namely $\mathrm{Ca} / \mathrm{Mg}$, glutathione, n-3 PUFA, Nacetylcysteine, AC591 and Guilongtongluofang. Apart from the interventions highlighted in the latest recommendations from American Society of Clinical Oncology (ASCO) and European Society for Medical Oncology (ESMO) $(7,8)$, this study included thirteen additional interventions. While the assessment of interventions in the "Insufficient Evidence" and "Not Recommended" categories were consistent with the guidelines, further six interventions identified as "Has Potential" warrant further investigation (Table 1).

While the pooled results from six RCTs showed that $\mathrm{Ca} / \mathrm{Mg}$ was associated with a statistically significant reduction in CTCAE grade 2 and above (Figure 3), this benefit was no longer observed following a sensitivity analysis that excluded two studies with early termination $(39,41)$ (Figure S2). In addition, the pooled results for studies that assessed outcome via OSS grade 2 and above neuropathy was not statistically significant (Figure S3). Hence, it is not possible to conclude from these results that $\mathrm{Ca} /$ $\mathrm{Mg}$ is effective in preventing OIPN.

Three other pharmaceuticals, namely $\mathrm{N}$-acetylcysteine, glutathione and n-3 PUFA, appeared to have the potential to prevent OIPN. While $\mathrm{N}$-acetylcysteine was associated with a statistically significant reduction in CTCAE grade 2 and above neuropathy based on pooled results from two trials $(46,47)$ (Figure 4), the overall small sample size and lack of blinding of one trial limited the overall certainty of the result. For glutathione, as compared to the results from older and smaller studies included in the previous Cochrane meta-analysis (80), this analysis pooled results from three recent trials $(30,42,43)$ (Figure 5), and showed possible benefit with high GRADE certainty. Nonetheless, the selected trials did not fully address existing concerns over glutathione's effect on oxaliplatin's clinical efficacy by providing cancer-related outcomes such as overall survival and did not assess the optimal dosage of glutathione. Also, N-3 PUFA was shown by Esfahani et al. (45) to be effective 
in preventing OIPN when measured in terms of Total Neuropathy Score but further studies are needed to affirm the result given limitations of the study explained in the results section.

In the herbal medicines examined, AC591 and Guilongtongluofang were associated with statistically significant reduction in the risk of $\operatorname{OIPN}(37,44)$. AC591, also known as Huangqi Guizhi Wuwu decoction, is a fixed compound extracted from five Traditional Chinese Medicine (TCM) herbs, while Guilongtongluofang is a concoction extracted from ten TCM herbs. A network meta-analysis on the efficacy of TCM in preventing OIPN also supported the neuroprotective benefit of Astragalus membranaceus, the main component in both AC591 and Guilongtongluofang (81). However, there is little evidence on the mechanism, safety profile, and effectiveness of these two compounds when considering the latest oncology guidelines for CIPN $(7,8)$.

The strengths of this study include the comprehensive coverage of interventions studied between 2005 and 2020, the strict selection criteria for only prospective RCTs where the patients were naïve to neurotoxic chemotherapy, the specificity for chronic OIPN which is distinct from peripheral neuropathy associated with other chemotherapy agents such as cisplatin or paclitaxel, and the holistic rating of interventions using a combination of RR, GRADE score, and risk of bias assessment. A network meta-analysis was also conducted to derive indirect results of the effect of intervention against placebo from studies that compared between two types of interventions.

This study has several limitations common to most metaanalyses. Firstly, it used summary data rather than individual patient data, which limits the ability of the study to control for confounders between trials. Secondly, analysis could only be conducted on trials that were published and searchable via English-based databases, which introduces an inherent publication bias. This was mitigated by including conference abstracts in the literature search. Finally, the lack of a standardized assessment method for OIPN limits comparison across trials (82).

\section{Pre-Clinical Studies and Future Directions}

Given the lack of definitive evidence on preventive interventions for OIPN, it is paramount to examine the relevant theoretical background and pre-clinical studies, so as to guide future trials.

Mechanisms for OIPN can be classified into platinum-related or oxaliplatin-specific. Platinum-related mechanisms include direct platinum-induced DNA damage in dorsal root ganglion, mitochondrial dysfunction, oxidative stress, and increased neuronal apoptosis $(10,83)$. On the other hand, oxaliplatinspecific mechanisms and targeted neuroprotective interventions have been examined in various pre-clinical studies, as summarized in a recent systematic review by Kawashiri et al. (84). Unfortunately, success in animal-based models has not consistently translated into clinical effectiveness. Various agents including anti-oxidants have been shown to reduce oxaliplatininduced behavior alterations in mice (85), but when assessed in clinical trials, they range from being not recommended [e.g. Vitamin $\mathrm{E}(\alpha$-tocopherol)] to requiring further evidence to validate neuroprotective potential (e.g. glutathione and n-3 PUFA). Nonetheless, there are many emerging therapeutic targets worthy of further clinical assessment. Notable mechanisms in pre-clinical studies include remediation of oxaliplatin-induced peroxisome alterations by activating Peroxisome Proliferator Activated Receptor- $\gamma$ (PPAR- $\gamma$ ) (86), antagonism of noxious hypersensitivity mediated by interactions between astrocytic vascular endothelial growth factor-A (VEGF-A) and VEGF receptor-1 (87), and alternatively via the $\alpha 9 \alpha 10$ nicotinic acetylcholine receptors (nAChR) (88), and reduction of neuronal inflammation by promoting Sphingosine 1-Phosphate Receptor $2\left(\mathrm{~S}_{1} \mathrm{P}_{2}\right)$ signaling (89).

Lastly, a paucity and need for more studies on nonpharmacological interventions was noted. Only one trial, exploring air wave pressure in preventing OIPN, fulfilled the selection criteria (59), however it was unable to demonstrate any significant benefit. In other CIPN prevention studies, four main non-pharmacological interventions have previously been assessed in RCTs, namely acupuncture (17), exercise $(18,90)$, compression therapy (91), and cryotherapy (15, 16, 19-21, 92, 93). Of these, the most promising intervention has been cryotherapy $(7,8)$. In a recent meta-analysis on the effectiveness of cryotherapy by Bailey et al. (94), it was noted that all seven trials assessed taxane-induced peripheral neuropathy, although the trial by Beijers et al. also recruited patients on oxaliplatin (21). Given that cold exposure is generally avoided to prevent acute OIPN, it is interesting that this study explored cryotherapy for CIPN prevention including an oxaliplatin cohort. One-third of the patients in this trial discontinued the intervention due to intolerance, however further details are unavailable. This preliminary evidence suggests future studies can be conducted explore cryotherapy for OIPN prevention.

\section{CONCLUSIONS}

In summary, despite the numerous pharmacological interventions reported in trials in recent years, there is insufficient evidence of any clinical success in OIPN prevention. Given the encouraging results seen in pre-clinical studies on OIPN prevention and emergence of nonpharmacological interventions in CIPN prevention, future studies could explore their potential. Moreover, studies with adequate recruitment, randomization and blinding, and standardized reporting of outcomes are necessary.

\section{DATA AVAILABILITY STATEMENT}

The data analyzed in this study is subject to the following licenses/restrictions: Readers may contact the authors for access to our datasets. Requests to access these datasets should be directed to siyu.peng@mohh.com.sg. 


\section{AUTHOR CONTRIBUTIONS}

RS, YS, and $A B$ conceived the research question and supervised the study. SP and AY planned the study methodology, performed data collection, analyzed results, and drafted the manuscript. SP performed statistical analysis with advice from YS. NC drafted and registered the study with the PROSPERO database. All authors provided critical feedback and helped shape the discussion. All authors contributed to the article and approved the submitted version.

\section{FUNDING}

This work was supported by the National Medical Research Council under its Clinician Scientist - Individual Research Grant (NMRC/CNIG/1167/2017); the National University Health

\section{REFERENCES}

1. Keum N, Giovannucci E. Global Burden of Colorectal Cancer: Emerging Trends, Risk Factors and Prevention Strategies. Nat Rev Gastroenterol Hepatol (2019) 16(12):713-32. doi: 10.1038/s41575-019-0189-8

2. Andre T, Boni C, Navarro M, Tabernero J, Hickish T, Topham C, et al. Improved Overall Survival With Oxaliplatin, Fluorouracil, and Leucovorin as Adjuvant Treatment in Stage II or III Colon Cancer in the MOSAIC Trial. J Clin Oncol (2009) 27(19):3109-16. doi: 10.1200/ JCO.2008.20.6771

3. Loprinzi CL, Qin R, Dakhil SR, Fehrenbacher L, Flynn KA, Atherton P, et al. Phase III Randomized, Placebo-Controlled, Double-Blind Study of Intravenous Calcium and Magnesium to Prevent Oxaliplatin-Induced Sensory Neurotoxicity (N08CB/Alliance). J Clin Oncol (2014) 32(10):9971005. doi: 10.1200/JCO.2013.52.0536

4. Park SB, Lin CSY, Krishnan AV, Goldstein D, Friedlander ML, Kiernan MC. Long-Term Neuropathy After Oxaliplatin Treatment: Challenging the Dictum of Reversibility. Oncologist (2011) 16(5):708-16. doi: 10.1634/ theoncologist.2010-0248

5. Pietrangeli A, Leandri M, Terzoli E, Jandolo B, Garufi C. Persistence of HighDose Oxaliplatin-Induced Neuropathy at Long-Term Follow-Up. Eur Neurol (2006) 56(1):13-6. doi: 10.1159/000094376

6. Pachman DR, Qin R, Seisler DK, Smith EML, Beutler AS, Ta LE, et al. Clinical Course of Oxaliplatin-Induced Neuropathy: Results From the Randomized Phase III Trial N08CB (Alliance). J Clin Oncol (2015) 33(30):3416-22. doi: 10.1200/JCO.2014.58.8533

7. Loprinzi CL, Lacchetti C, Bleeker J, Cavaletti G, Chauhan C, Hertz DL, et al. Prevention and Management of Chemotherapy-Induced Peripheral Neuropathy in Survivors of Adult Cancers: ASCO Guideline Update. J Clin Oncol (2020) 38(28):3325-48. doi: 10.1200/JCO.20.01399

8. Jordan B, Margulies A, Cardoso F, Cavaletti G, Haugnes HS, Jahn P, et al. Systemic Anticancer Therapy-Induced Peripheral and Central Neurotoxicity: ESMO- EONS -EANO Clinical Practice Guidelines for Diagnosis, Prevention, Treatment and Follow-Up. Ann Oncol (2020) 31(10):1306-19. doi: 10.1016/ j.annonc.2020.07.003

9. Park SB, Lin CSY, Krishnan AV, Goldstein D, Friedlander ML, Kiernan MC. Dose Effects of Oxaliplatin on Persistent and Transient Na+ Conductances and the Development of Neurotoxicity. PloS One (2011) 6(4):e18469. doi: 10.1371/journal.pone.0018469

10. Kanat O, Ertas H, Caner B. Platinum-Induced Neurotoxicity: A Review of Possible Mechanisms. World J Clin Oncol (2017) 8(4):329. doi: 10.5306/ wjco.v8.i4.329

11. Ohsawa M, Otake S, Murakami T, Yamamoto S, Makino T, Ono H. Gabapentin Prevents Oxaliplatin-Induced Mechanical Hyperalgesia in Mice. J Pharmacol Sci (2014) 125(3):292-9. doi: 10.1254/jphs.14058FP
System under its NUHS Summit Research Program - Cancer (NCSP N-171-000-493-001); National University of Singapore under its N.1 Institute for Health's Translational Core.

\section{ACKNOWLEDGMENTS}

We thank Prof Charles L. Loprinzi (MD) for providing requested trial data and advising on the discussion of the paper.

\section{SUPPLEMENTARY MATERIAL}

The Supplementary Material for this article can be found online at: https://www.frontiersin.org/articles/10.3389/fonc.2022. 731223/full\#supplementary-material

12. Lee M, Cho S, Roh K, Chae J, Park JH, Park J, et al. Glutathione Alleviated Peripheral Neuropathy in Oxaliplatin-Treated Mice by Removing Aluminum From Dorsal Root Ganglia. Am J Transl Res (2017) 9(3):926-39.

13. Mizuno K, Shibata K, Komatsu R, Omiya Y, Kase Y, Koizumi S. An Effective Therapeutic Approach for Oxaliplatin-Induced Peripheral Neuropathy Using a Combination Therapy With Goshajinkigan and Bushi. Cancer Biol Ther (2016) 17(11):1206-12. doi: 10.1080/15384047.2016.1210742

14. Greco T, Zangrillo A, Biondi-Zoccai G, Landoni G. Meta-Analysis: Pitfalls and Hints. Heart Lung Vessel (2013) 5(4):219-25.

15. Sundar R, Bandla A, Tan SSH, Liao L-D, Kumarakulasinghe NB, Jeyasekharan $\mathrm{AD}$, et al. Limb Hypothermia for Preventing Paclitaxel-Induced Peripheral Neuropathy in Breast Cancer Patients: A Pilot Study. Front Oncol (2017) 6 (274). doi: 10.3389/fonc.2016.00274

16. Hanai A, Ishiguro H, Sozu T, Tsuda M, Yano I, Nakagawa T, et al. Effects of Cryotherapy on Objective and Subjective Symptoms of Paclitaxel-Induced Neuropathy: Prospective Self-Controlled Trial. JNCI: J Natl Cancer Institute (2018) 110(2):141-8. doi: 10.1093/jnci/djx178

17. Greenlee H, Crew KD, Capodice J, Awad D, Buono D, Shi Z, et al. Randomized Sham-Controlled Pilot Trial of Weekly Electro-Acupuncture for the Prevention of Taxane-Induced Peripheral Neuropathy in Women With Early Stage Breast Cancer. Breast Cancer Res Treat (2016) 156(3):45364. doi: 10.1007/s10549-016-3759-2

18. Kleckner IR, Kamen C, Gewandter JS, Mohile NA, Heckler CE, Culakova E, et al. Effects of Exercise During Chemotherapy on Chemotherapy-Induced Peripheral Neuropathy: A Multicenter, Randomized Controlled Trial. Support Care Cancer (2018) 26(4):1019-28. doi: 10.1007/s00520-017-4013-0

19. Eckhoff L, Knoop AS, Jensen MB, Ejlertsen B, Ewertz M. Risk of DocetaxelInduced Peripheral Neuropathy Among 1,725 Danish Patients With Early Stage Breast Cancer. Breast Cancer Res Treat (2013) 142(1):109-18. doi: 10.1007/s10549-013-2728-2

20. Ruddy KJ, Le-Rademacher J, Lacouture ME, Wilkinson M, Onitilo AA, Vander Woude AC, et al. Randomized Controlled Trial of Cryotherapy to Prevent Paclitaxel-Induced Peripheral Neuropathy (RU221511I); an ACCRU Trial. Breast (2019) 48:89-97. doi: 10.1016/j.breast.2019.09.011

21. Beijers AJM, Bonhof CS, Mols F, Ophorst J, de Vos-Geelen J, Jacobs EMG, et al. Multicenter Randomized Controlled Trial to Evaluate the Efficacy and Tolerability of Frozen Gloves for the Prevention of Chemotherapy-Induced Peripheral Neuropathy. Ann Oncol (2020) 31(1):131-6. doi: 10.1016/j.annonc.2019.09.006

22. Jordan B, Jahn F, Beckmann J, Unverzagt S, Müller-Tidow C, Jordan K Calcium and Magnesium Infusions for the Prevention of Oxaliplatin-Induced Peripheral Neurotoxicity: A Systematic Review. Oncology (2016) 90(6):299306. doi: 10.1159/000445977

23. Kuriyama A, Endo K. Goshajinkigan for Prevention of ChemotherapyInduced Peripheral Neuropathy: A Systematic Review and Meta-Analysis. Support Care Cancer (2018) 26(4):1051-9. doi: 10.1007/s00520-017-4028-6 
24. Zhang AC, De Silva MEH, MacIsaac RJ, Roberts L, Kamel J, Craig JP, et al. Omega-3 Polyunsaturated Fatty Acid Oral Supplements for Improving Peripheral Nerve Health: A Systematic Review and Meta-Analysis. Nutr Rev (2020) 78(4):323-41. doi: 10.1093/nutrit/nuz054

25. Ewertz M, Qvortrup C, Eckhoff L. Chemotherapy-Induced Peripheral Neuropathy in Patients Treated With Taxanes and Platinum Derivatives. Acta Oncol (2015) 54(5):587-91. doi: 10.3109/0284186X.2014.995775

26. Imai S, Koyanagi M, Azimi Z, Nakazato Y, Matsumoto M, Ogihara T, et al. Taxanes and Platinum Derivatives Impair Schwann Cells via Distinct Mechanisms. Sci Rep (2017) 7(1):5947. doi: 10.1038/s41598-017-05784-1

27. Moher D, Liberati A, Tetzlaff J, Altman DG. Preferred Reporting Items for Systematic Reviews and Meta-Analyses: The PRISMA Statement. J Clin Epidemiol (2009) 62(10):1006-12. doi: 10.1016/j.jclinepi.2009.06.005

28. Schünemann H BJ, Guyatt G, Oxman A. GRADE Handbook for Grading Quality of Evidence and Strength of Recommendations. The GRADE Working Group (2013). 2013.

29. Higgins JPT, Thomas J, Chandler J, Cumpston M, Page T, Welch MJ, editors Cochrane Handbook for Systematic Reviews of Interventions version 6.2 (updated February 2021). In: Cochrane. Chichester (UK): John Wiley \& Sons (2021). Available at: www.training.cochrane.org/handbook.

30. Dong M, Xing P-y, Liu P, Feng F-y, Shi Y-k. Assessment of the Protective Effect of Calcium-Magnesium Infusion and Glutathione on OxaliplatinInduced Neurotoxicity. Zhonghua Zhong Liu Za Zhi (2010) 32(3):208-11.

31. Zimmer P, Trebing S, Timmers-Trebing U, Schenk A, Paust R, Bloch W, et al. Eight-Week, Multimodal Exercise Counteracts a Progress of ChemotherapyInduced Peripheral Neuropathy and Improves Balance and Strength in Metastasized Colorectal Cancer Patients: A Randomized Controlled Trial. Support Care Cancer (2018) 26(2):615-24. doi: 10.1007/s00520-017-3875-5

32. Guo Y, Jones D, Palmer JL, Forman A, Dakhil SR, Velasco MR, et al. Oral Alpha-Lipoic Acid to Prevent Chemotherapy-Induced Peripheral Neuropathy: A Randomized, Double-Blind, Placebo-Controlled Trial. Support Care Cancer (2014) 22(5):1223-31. doi: 10.1007/s00520-013-2075-1

33. Kottschade LA, Sloan JA, Mazurczak MA, Johnson DB, Murphy BP, Rowland $\mathrm{KM}$, et al. The Use of Vitamin $\mathrm{E}$ for the Prevention of Chemotherapy-Induced Peripheral Neuropathy: Results of a Randomized Phase III Clinical Trial. Support Care Cancer (2011) 19(11):1769-77. doi: 10.1007/s00520-010-1018-3

34. Kautio AL, Haanpaa M, Leminen A, Kalso E, Kautiainen H, Saarto T. Amitriptyline in the Prevention of Chemotherapy-Induced Neuropathic Symptoms. Anticancer Res (2009) 29(7):2601-6.

35. Nihei S, Sato J, Kashiwaba M, Itabashi T, Kudo K, Takahashi K. Efficacy and Safety of Pregabalin for Oxaliplatin- and Paclitaxel-Induced Peripheral Neuropathy. Gan To Kagaku Ryoho (2013) 40(9):1189-93.

36. Schloss JM, Colosimo M, Airey C, Masci P, Linnane AW, Vitetta L. A Randomised, Placebo-Controlled Trial Assessing the Efficacy of an Oral B Group Vitamin in Preventing the Development of Chemotherapy-Induced Peripheral Neuropathy (CIPN). Support Care Cancer (2017) 25(1):195-204. doi: 10.1007/s00520-016-3404-y

37. Cheng X, Huo J, Wang D, Cai X, Sun X, Lu W, et al. Herbal Medicine AC591 Prevents Oxaliplatin-Induced Peripheral Neuropathy in Animal Model and Cancer Patients. Front Pharmacol (2017) 8:344. doi: 10.3389/ fphar.2017.00344

38. Kiladze IZ, Sharikadze N, Esakia T. Calcium Gluconate and Magnesium Sulfate in Preventing Neurotoxicity in Patients Receiving Oxaliplatin-Based Combination chemotherapy-capeOX. Ann Oncol (2016) 27:vi166. doi: 10.1093/annonc/ mdw370.52

39. Ishibashi K, Okada N, Miyazaki T, Sano M, Ishida H. Effect of Calcium and Magnesium on Neurotoxicity and Blood Platinum Concentrations in Patients Receiving Mfolfox6 Therapy: A Prospective Randomized Study. Int J Clin Oncol (2010) 15(1):82-7. doi: 10.1007/s10147-009-0015-3

40. Gobran NS. Role of Calcium and Magnesium Infusion in Prevention of Oxaliplatin Neurotoxicity. A Phase III Trial. Chinese German J Clin Oncol (2013) 12(5):232-6. doi: 10.1007/s10330-013-1161-7

41. Grothey A, Nikcevich DA, Sloan JA, Kugler JW, Silberstein PT, Dentchev T, et al. Intravenous Calcium and Magnesium for Oxaliplatin-Induced Sensory Neurotoxicity in Adjuvant Colon Cancer: NCCTG N04c7. J Clin Oncol (2011) 29(4):421-7. doi: 10.1200/JCO.2010.31.5911

42. Milla P, Airoldi M, Weber G, Drescher A, Jaehde U, Cattel L. Administration of Reduced Glutathione in FOLFOX4 Adjuvant Treatment for Colorectal
Cancer: Effect on Oxaliplatin Pharmacokinetics, Pt-DNA Adduct Formation, and Neurotoxicity. Anticancer Drugs (2009) 20(5):396-402. doi: 10.1097/ CAD.0b013e32832a2dc1

43. Pang DM, Deng YM, Lan XS, Feng WN, Liang JM, Xu YH. Efficacy of Reduced Glutathione in Preventing and Reducing Neurotoxicity of Oxaliplatin. Chin J Cancer Prev Treat (2010) 17(24):2057-9+69. doi: 10.16073/j.cnki .cjcpt.2010.24.016

44. Liu Y, Zhu G, Han L, Liu J, Ma T, Yu H. Clinical Study on the Prevention of Oxaliplatin-Induced Neurotoxicity With Guilongtongluofang: Results of a Randomized, Double-Blind, Placebo-Controlled Trial. Evid Based Complement Alternat Med (2013) 2013:541217. doi: 10.1155/2013/541217

45. Esfahani A, Somi MH, Ayromlou H, Nikanfar A, Jafarabadi MA, Sadat BE, et al. The Effect of N-3 Polyunsaturated Fatty Acids on Incidence and Severity of Oxaliplatin Induced Peripheral Neuropathy: A Randomized Controlled Trial. biomark Res (2016) 4:13. doi: 10.1186/s40364-016-0066-3

46. Lin P-C, Lee M-Y, Wang W-S, Yen C-C, Chao T-C, Hsiao L-T, et al. NAcetylcysteine has Neuroprotective Effects Against Oxaliplatin-Based Adjuvant Chemotherapy in Colon Cancer Patients: Preliminary Data. Support Care Cancer (2006) 14(5):484-7. doi: 10.1007/s00520-006-0018-9

47. Bondad N, Boostani R, Barri A, Elyasi S, Allahyari A. Protective Effect of N-Acetylcysteine on Oxaliplatin-Induced Neurotoxicity in Patients With Colorectal and Gastric Cancers: A Randomized, Double Blind, PlaceboControlled, Clinical Trial. J Oncol Pharm Pract (2020) 26(7):1575-82. doi: $10.1177 / 1078155219900788$

48. von Delius S, Eckel F, Wagenpfeil S, Mayr M, Stock K, Kullmann F, et al. Carbamazepine for Prevention of Oxaliplatin-Related Neurotoxicity in Patients With Advanced Colorectal Cancer: Final Results of a Randomised, Controlled, Multicenter Phase II Study. Invest New Drugs (2007) 25(2):173-80. doi: 10.1007/ s10637-006-9010-y

49. Wang W-S, Lin J-K, Lin T-C, Chen W-S, Jiang J-K, Wang H-S, et al. Oral Glutamine Is Effective for Preventing Oxaliplatin-Induced Neuropathy in Colorectal Cancer Patients. Oncologist (2007) 12(3):312-9. doi: 10.1634/ theoncologist.12-3-312

50. Saad AST, Yehia RM, Mostafa SS, Elabhar HS, Schaalan MF. L-Carnosine for Prevention of Oxaliplatin-Induced Neuropathy in Colorectal Cancer Patients (Pts). Ann Oncol (2016) 27:vi508. doi: 10.1093/annonc/ mdw390.35

51. Yehia R, Saleh S, El Abhar H, Saad AS, Schaalan M. L-Carnosine Protects Against Oxaliplatin-Induced Peripheral Neuropathy in Colorectal Cancer Patients: A Perspective on Targeting Nrf-2 and NF-kb Pathways. Toxicol Appl Pharmacol (2019) 365:41-50. doi: 10.1016/j.taap.2018.12.015

52. El-Fatatry BM, Ibrahim OM, Hussien FZ, Mostafa TM. Role of Metformin in Oxaliplatin-Induced Peripheral Neuropathy in Patients With Stage III Colorectal Cancer: Randomized, Controlled Study. Int J Colorectal Dis (2018) 33(12):1675-83. doi: 10.1007/s00384-018-3104-9

53. Zhang RX, Lu ZH, Wan DS, Wu XJ, Ding PR, Kong LH, et al. Neuroprotective Effect of Neurotropin on Chronic Oxaliplatin-Induced Neurotoxicity in Stage II and Stage III Colorectal Cancer Patients: Results From a Prospective, Randomised, Single-Centre, Pilot Clinical Trial. Int J Colorectal Dis (2012) 27 (12):1645-50. doi: 10.1007/s00384-012-1509-4

54. Motoo Y, Tomita Y, Fujita H. Prophylactic Efficacy of Ninjin'yoeito for Oxaliplatin-Induced Cumulative Peripheral Neuropathy in Patients With Colorectal Cancer Receiving Postoperative Adjuvant Chemotherapy: A Randomized, Open-Label, Phase 2 Trial (HOPE-2). Int J Clin Oncol (2020) 25(6):1123-9. doi: 10.1007/s10147-020-01648-3

55. Argyriou AA, Chroni E, Polychronopoulos P, Iconomou G, Koutras A, Makatsoris T, et al. Efficacy of Oxcarbazepine for Prophylaxis Against Cumulative Oxaliplatin-Induced Neuropathy. Neurology (2006) 67 (12):2253-5. doi: 10.1212/01.wnl.0000249344.99671.d4

56. de Andrade DC, Teixeira MJ, Galhardoni R, Ferreira KSL, Mileno PB, Scisci $\mathrm{N}$, et al. Pregabalin for the Prevention of Oxaliplatin-Induced Painful Neuropathy: A Randomized, Double-Blind Trial. Oncologist (2017) 22 (10):1154-E105. doi: 10.1634/theoncologist.2017-0235

57. Song M, Li L, Chang ZW, Meng Y, Chang J. Assessment of the Protective Effect of Venlafaxine on Oxaliplatin-Induced Neurotoxicity. Chin J Cancer Prev Treat (2013) 20(7):546-9. doi: 10.16073/j.cnki.cjcpt.2013.07.020

58. Zimmerman C, Atherton PJ, Pachman D, Seisler D, Wagner-Johnston N, Dakhil S, et al. MC11C4: A Pilot Randomized, Placebo-Controlled, Double- 
Blind Study of Venlafaxine to Prevent Oxaliplatin-Induced Neuropathy. Support Care Cancer (2016) 24(3):1071-8. doi: 10.1007/s00520-015-2876-5

59. Qian W, Pan Y, Yang Y. Observe the Efficacy of the Air Wave Pressure Therapeutic Equipment in Treatment of Oxaliplatin Nerve Toxicity. Chinese German J Clin Oncol (2012) 11(10):602-4. doi: 10.1007/s10330-012-1073-y

60. Gado AM, El-Mashad W, El-Mashad N. Role of Alpha Lipoic Acid in Prevention of Oxaliplatin Neurological Toxicity. Life Sci J (2013) 10(4):3739. doi: $10.7537 /$ marslsj100413.49

61. Lu P, Fan QX, Wang LX, Wang X, Zong H, Wang RL. Prophylactic Effect of Amifostine on Oxaliplatin-Related Neurotoxicity in Patients With Digestive Tract Tumors. Ai Zheng (2008) 27(10):1117-20.

62. Uchida Y, Kusakawa G, Sakai T, Takamoto M, Kimoto S, Takemasa S. Prevention of Oxaliplatin-Induced Peripheral Neuropathy (OIPN): Randomized Placebocontrolled Trial Results Investigating Efficacy and Safety of a Recombinant Soluble Human Thrombomodulin (ART-123). Supportive Care Cancer (2019) 27(1):S13-S4. doi: 10.1007/s00520-01904813-1

63. Glimelius B, Manojlovic N, Pfeiffer P, Mosidze B, Kurteva G, Karlberg M, et al. Persistent Prevention of Oxaliplatin-Induced Peripheral Neuropathy Using Calmangafodipir (PledOx $\left({ }^{\circledR}\right)$ ): A Placebo-Controlled Randomised Phase II Study (PLIANT). Acta Oncol (2018) 57(3):393-402. doi: 10.1080/ 0284186X.2017.1398836

64. Kobayashi M, Sato R, Komura T, Ichikawa H, Hirashima T, Otake S, et al. Protective Effect of the Oral Administration of Cystine and Theanine on Oxaliplatin-Induced Peripheral Neuropathy: A Pilot Randomized Trial. Int $J$ Clin Oncol (2020) 25(10):1814-21. doi: 10.1007/s10147-020-01728-4

65. Samson B, Dube P, Aucoin N, Wierzbicki R, Maroun J, Letourneau R, et al. Efficacy of Glutamine Added to Calcium/Magnesium on OxaliplatinInduced Peripheral Sensory Neuropathy in Patients With Colorectal Cancer, a Phase III Study. Eur J Cancer (2013) 49:S518-9. doi: 10.1016/ S0959-8049(13)70063-7

66. Wang D-S, Wang Z-Q, Chen G, Peng J-W, Wang W, Deng Y-H, et al. Phase III Randomized, Placebo-Controlled, Double-Blind Study of Monosialotetrahexosylganglioside for the Prevention of Oxaliplatin-Induced Peripheral Neurotoxicity in Stage II/III Colorectal Cancer. Cancer Med (2019) 9(1):151-9. doi: 10.1002/cam4.2693

67. Oki E, Emi Y, Kojima H, Higashijima J, Kato T, Miyake Y, et al. Preventive Effect of Goshajinkigan on Peripheral Neurotoxicity of FOLFOX Therapy (GENIUS Trial): A Placebo-Controlled, Double-Blind, Randomized Phase III Study. Int J Clin Oncol (2015) 20(4):767-75. doi: 10.1007/s10147-015-0784-9

68. Kono T, Hata T, Morita S, Munemoto Y, Matsui T, Kojima H, et al. Goshajinkigan Oxaliplatin Neurotoxicity Evaluation (GONE): A Phase 2, Multicenter, Randomized, Doubleblind, Placebocontrolled Trial of Goshajinkigan to Prevent Oxaliplatininduced Neuropathy. Cancer Chemother Pharmacol (2013) 72(6):1283-90. doi: 10.1007/s00280-013-2306-7

69. Wang XS, Shi Q, Bhadkamkar NA, Cleeland CS, Garcia-Gonzalez A, Aguilar JR, et al. Minocycline for Symptom Reduction During Oxaliplatin-Based Chemotherapy for Colorectal Cancer: A Phase II Randomized Clinical Trial. J Pain Symptom Manage (2019) 58(4):662-71. doi: 10.1016/j.jpainsymman.2019.06.018

70. Bruna J, Videla S, Argyriou AA, Velasco R, Villoria J, Santos C, et al. Efficacy of a Novel Sigma-1 Receptor Antagonist for Oxaliplatin-Induced Neuropathy: A Randomized, Double-Blind, Placebo-Controlled Phase IIa Clinical Trial. Neurotherapeutics (2018) 15(1):178-89. doi: 10.1007/s13311-017-0572-5

71. Trinh T, Park SB, Murray J, Pickering H, Lin CSY, Martin A, et al. NeuHorizons: Neuroprotection and Therapeutic Use of Riluzole for the Prevention of Oxaliplatin-Induced Neuropathy-A Randomised Controlled Trial. Supportive Care Cancer (2020) 29(2):1103-10. doi: 10.1007/s00520-02005591-x

72. Xu K, Cheng W-t, Hu Z-w, Wang S. Effect of Tanshinone IIA in Preventing and Treating Oxaliplatin Induced Peripheral Neuropathy. Zhongguo Zhong Xi Yi Jie He Za Zhi (2016) 36(5):559-63. doi: 10.7661/cjim.2016.05.0559

73. Salehi Z, Roayaei M. Effect of Vitamin E on Oxaliplatin-Induced Peripheral Neuropathy Prevention: A Randomized Controlled Trial. Int J Prev Med (2015) 6:104. doi: 10.4103/2008-7802.169021

74. de Afonseca SO, Cruz FM, Cubero D, Lera AT, Schindler F, Okawara M, et al. Vitamin E for Prevention of Oxaliplatin-Induced Peripheral Neuropathy: A Pilot Randomized Clinical Trial. Sao Paulo Med J (2013) 131(1):35-8. doi: 10.1590/S1516-31802013000100006
75. Chay W-Y, Tan S-H, Lo Y-L, Ong SY-K, Ng H-C, Gao F, et al. Use of Calcium and Magnesium Infusions in Prevention of Oxaliplatin Induced Sensory Neuropathy. Asia Pac J Clin Oncol (2010) 6(4):270-7. doi: 10.1111/j.1743-7563.2010.01344.x

76. Hochster HS, Grothey A, Childs BH. Use of Calcium and Magnesium Salts to Reduce Oxaliplatin-Related Neurotoxicity. J Clin Oncol (2007) 25(25):4028-9. doi: 10.1200/JCO.2007.13.5251

77. Zhu Y, Yang J, Jiao S, Ji T. Ganglioside-Monosialic Acid (GM1) Prevents Oxaliplatin-Induced Peripheral Neurotoxicity in Patients With Gastrointestinal Tumors. World J Surg Oncol (2013) 11:19. doi: 10.1186/1477-7819-11-19

78. Yazici O, Titiz AP, Ozdemir N, Aksoy S, Sendur MA, Arli B, et al. Role of Glutamine for Preventing Oxaliplatin Induced Peripheral Neuropathy (GELUPO): Results of Randomized Open-Label Phase II Trial. Ann Oncol (2016) 27:vi169. doi: 10.1093/annonc/mdw370.62

79. Nishioka M, Shimada M, Kurita N, Iwata T, Morimoto S, Yoshikawa K, et al. The Kampo Medicine, Goshajinkigan, Prevents Neuropathy in Patients Treated by FOLFOX Regimen. Int J Clin Oncol (2011) 16(4):322-7. doi: 10.1007/s10147-010-0183-1

80. Albers JW, Chaudhry V, Cavaletti G, Donehower RC. Interventions for Preventing Neuropathy Caused by Cisplatin and Related Compounds. Cochrane Database Syst $\operatorname{Rev}$ (2014) (3):Cd005228. doi: 10.1002/14651858.CD005228.pub4

81. Wei XC-Z LQ, Wang H, Wang CG, Deng Q, Li X. Efficacy of Traditional Chinese Medicines in Preventing Oxaliplatin-Induced Peripheral Neurotoxicity in Cancer Patients: A Network Meta-Analysis. Chin Herbal Medicines (2017) 9(2):161-8. doi: 10.1016/S1674-6384(17)60090-X

82. Cavaletti G. Chemotherapy-Induced Peripheral Neurotoxicity: How can We Improve Knowledge? Lancet Oncol (2009) 10(6):539-40. doi: 10.1016/S14702045(09)70105-6

83. Cavaletti G, Marmiroli P. Chemotherapy-Induced Peripheral Neurotoxicity. Nat Rev Neurol (2010) 6(12):657-66. doi: 10.1038/nrneurol.2010.160

84. Kawashiri T, Mine K, Kobayashi D, Inoue M, Ushio S, Uchida M, et al. Therapeutic Agents for Oxaliplatin-Induced Peripheral Neuropathy; Experimental and Clinical Evidence. Int J Mol Sci (2021) 22(3):1393. doi: 10.3390/ijms22031393

85. Di Cesare Mannelli L, Zanardelli M, Failli P, Ghelardini C. OxaliplatinInduced Neuropathy: Oxidative Stress as Pathological Mechanism. Protective Effect of Silibinin. J Pain (2012) 13(3):276-84. doi: 10.1016/j.jpain.2011.11.009

86. Zanardelli M, Micheli L, Cinci L, Failli P, Ghelardini C, Di Cesare Mannelli L. Oxaliplatin Neurotoxicity Involves Peroxisome Alterations. Ppary Agonism as Preventive Pharmacological Approach. PloS One (2014) 9(7):e102758. doi: 10.1371/journal.pone.0102758

87. Micheli L, Parisio C, Lucarini E, Vona A, Toti A, Pacini A, et al. VEGF-A VEGFR-1 Signalling and Chemotherapy-Induced Neuropathic Pain: Therapeutic Potential of a Novel Anti-VEGFR-1 Monoclonal Antibody. J Exp Clin Cancer Res (2021) 40(1):1. doi: 10.1186/s13046-021-02127-x

88. Pacini A, Micheli L, Maresca M, Branca JJV, McIntosh JM, Ghelardini C, et al. The $\alpha 9 \alpha 10$ Nicotinic Receptor Antagonist $\alpha$-Conotoxin RgIA Prevents Neuropathic Pain Induced by Oxaliplatin Treatment. Exp Neurol (2016) 282:37-48. doi: 10.1016/j.expneurol.2016.04.022

89. Wang W, Xiang P, Chew WS, Torta F, Bandla A, Lopez V, et al. Activation of Sphingosine 1-Phosphate Receptor 2 Attenuates Chemotherapy-Induced Neuropathy. J Biol Chem (2020) 295(4):1143-52. doi: 10.1016/S0021-9258(17)49922-4

90. Streckmann F, Balke M, Lehmann HC, Rustler V, Koliamitra C, Elter T, et al. The Preventive Effect of Sensorimotor- and Vibration Exercises on the Onset of Oxaliplatin- or Vinca-Alkaloid Induced Peripheral Neuropathies - STOP. BMC Cancer (2018) 18(1):62. doi: 10.1186/s12885-017-3866-4

91. Tsuyuki S, Senda N, Kanng Y, Yamaguchi A, Yoshibayashi H, Kikawa Y, et al. Evaluation of the Effect of Compression Therapy Using Surgical Gloves on Nanoparticle Albumin-Bound Paclitaxel-Induced Peripheral Neuropathy: A Phase II Multicenter Study by the Kamigata Breast Cancer Study Group. Breast Cancer Res Treat (2016) 160(1):61-7. doi: 10.1007/s10549-016-3977-7

92. Griffiths C, Kwon N, Beaumont JL, Paice JA. Cold Therapy to Prevent Paclitaxel-Induced Peripheral Neuropathy. Support Care Cancer (2018) 26 (10):3461-9. doi: 10.1007/s00520-018-4199-9

93. Shigematsu H, Hirata T, Nishina M, Yasui D, Ozaki S. Cryotherapy for the Prevention of Weekly Paclitaxel-Induced Peripheral Adverse Events in Breast Cancer Patients. Support Care Cancer (2020) 28(10):5005-11. doi: 10.1007/ s00520-020-05345-9

94. Bailey AG, Brown JN, Hammond JM. Cryotherapy for the Prevention of Chemotherapy-Induced Peripheral Neuropathy: A Systematic Review. 
J Oncol Pharm Pract (2021) 27(1):156-64. doi: 10.1177/107815522 0959431

Conflict of Interest: RS is a member on the advisory board of Bristol Myers Squibb, Merck, Eisai, Bayer, Taiho, Novartis, MSD. He has received honoraria for talks from MSD, Eli Lilly, BMS, Roche, Taiho, Astra Zeneca. He has received travel funding from Paxman Coolers Ltd., Roche, Astra Zeneca, Taiho, Eisai. He has received research funding from Paxman Coolers, MSD. AB has received travel funding from Paxman Coolers Ltd.

The remaining authors declare that the research was conducted in the absence of any commercial or financial relationships that could be construed as a potential conflict of interest.
Publisher's Note: All claims expressed in this article are solely those of the authors and do not necessarily represent those of their affiliated organizations, or those of the publisher, the editors and the reviewers. Any product that may be evaluated in this article, or claim that may be made by its manufacturer, is not guaranteed or endorsed by the publisher.

Copyright (c) 2022 Peng, Ying, Chan, Sundar, Soon and Bandla. This is an open-access article distributed under the terms of the Creative Commons Attribution License (CC BY). The use, distribution or reproduction in other forums is permitted, provided the original author(s) and the copyright owner(s) are credited and that the original publication in this journal is cited, in accordance with accepted academic practice. No use, distribution or reproduction is permitted which does not comply with these terms. 